\title{
Towards Engineering Ontologies for Cognitive Profiling of Agents on the Semantic Web
}

\author{
Amna Basharat ${ }^{1}$, Gabriella Spinelli ${ }^{2}$ \\ ${ }^{1}$ National University of Computer and Emerging Sciences, Islamabad, Pakistan \\ amna.basharat@nu.edu.pk \\ ${ }^{2}$ Brunel University, West London, UK \\ gabriella.spinelli@,brunel.ac.uk
}

\begin{abstract}
Research shows that most agent-based collaborations suffer from lack of flexibility. This is due to the fact that most agent-based applications assume pre-defined knowledge of agents' capabilities and/or neglect basic cognitive and interactional requirements in multi-agent collaboration. The highlight of this paper is that it brings cognitive models (inspired from cognitive sciences and HCI) proposing architectural and knowledge-based requirements for agents to structure ontological models for cognitive profiling in order to increase cognitive awareness between themselves, which in turn promotes flexibility, reusability and predictability of agent behavior; thus contributing towards minimizing cognitive overload incurred on humans. The semantic web is used as an action mediating space, where shared knowledge base in the form of ontological models provides affordances for improving cognitive awareness.
\end{abstract}

\section{INTRODUCTION}

Agents are designed to continually act upon and monitor the Multi-Agent System (MAS) Environment, interacting with it and collaborating with other agents and entities, evaluating its state, and executing actions $[1,2,3,4]$. Research community is faced with the challenges of improving the limited visibility of agent's processing ability and behaviours that may be a result of possible mismatches between the agents' mental and implementation models. While a plethora of research done in recent years on collaborative and interoperable MAS, research reveals that there is still a long way to go before true homogenisation of agent communities can be achieved [5,6,8,13]. Recently, much effort has been expended on making agents interoperate in the emerging open environments and standards. Despite this effort, this goal has still not yet been achieved as Louis and Martinez [5,6 and 8] point out. This is because the variance of agent communication and functional pragmatics introduces a certain level of mismatch and the need of flexible and adaptive interactions that promote interoperation becomes imperative. This is particularly essential when the agents from diverse platforms intend to co-exist and cooperate in mutual.
Some interdisciplinary research has stressed the potential of cognitive models studied in cognitive science as substantial means of better probing multi-agent issues, by taking into account essential characteristics of cognitive agents and their various capacities [7]. Inspired by this very approach, this paper seeks to investigate the possibility of meeting the challenges of distributed multi-agent collaboration by bringing cognitive models and theories in the agent-mediated semantic web to achieve more robust and effective architectures that facilitate distributed collaboration- be it amongst agents or between agents and humans or vice versa. The interplay between theoretical approaches of Cognitive engineering and the agent-mediated semantic web is investigated in an attempt to improve the nature of multi-agent collaboration by promoting flexibility, reuse, interoperability and shared awareness based on heuristic reasoning. After a review of the available cognitive approaches that model human activities in interaction with any system (artefacts and the environment), the Action Cycle (AC) $[9,10,11]$ has been selected as the most promising approach for this research.

The cognitive perspective of the $\mathrm{AC}$ is adapted to provide a Cognitive Profiling Architecture for agents actions in a collaborative MAS environment. The architecture is intended to aid the designer in modelling the agent behaviour and action through a cognitive cycle. Using the principles of the proposed architecture designers can define both functional and non functional aspects of the design of an agent's interactive role in a collaborative scenario, especially focusing on the concepts of semantic and articulatory distances, as derived from $\mathrm{AC}$, as mismatch between agents' goals and its functional capabilities. Architectural and knowledge-based requirements for agents to structure ontological models for cognitive profiling in order to increase cognitive awareness amongst agents. By cognitive awareness, a term coined within this research, it is intended the ability of the web agents to diagnose their processing limitations and to establish interactions with the external environment (in the form of other agents including humans and software agents) using the principles derived from the AC. This is with the aim to support users' goals in a more direct manner by providing agents that can share, discover and access each other's capabilities in a collaborative manner and are able to function dynamically 
and adaptively without continuous human intervention. This brings about a more effective MAS environment; where agents may delegate each other tasks and goals based on each other's awareness of abilities, behaviours and affordances thus reducing the cognitive overload incurred on humans.

The rest of the paper is organized as follows: Section 2 describes the design goals and the architecture for cognitive profiling of agents, Section 3 details the Semantic Representation Model for the Cognitive Profiles of Agents. Section 4 describes the implementation and evaluation of the arechitecture. The use and rationale behind the architecture is described in Section 5 before presenting the Conclusions and directions for future work in Section 6.

\section{Architecture for Cognitive Profiling OF AGENTS}

\subsection{Design Goals for Cognitive Profiling of Agents}

The theoretical foundations of Cognitive Models such as the Action Cycle (AC) bring about important implications in the current semantic web architectures. Traditional webservice agent architectures only allow agents to discover about each others services. However this research claims that an architecture that is inspired from cognitive models would allow the agents to develop a cognitive awareness about each other which could bring to a more effective MAS environment. To validate this claim, a lower-level classification of design goals which provide the basis upon which the Cognitive Profiling Architecture is devised upon includes: (a) Enhanced Negotiation and Collaboration based on Cognitive Awareness (b) Flexibility and Reusability
(c)Adaptive Interaction and Interoperability (d)Discovery based on Heuristic reasoning and (e) Minimization of cognitive load on humans.

\subsection{High-Level Design of Agent Infrastructural Elements}

The agent is viewed as the primary entity that interacts and functions in a MAS environment. In MAS environment the fundamental unit of agent's social ability is its interaction with other agents using messages in Agent Communication Language (ACL) [21]. Agent's Interaction is modelled to have two stages: Execution and Evaluation, each with its respective steps being managed by Execution and Evaluation Manager respectively as shown in Figure 1. The Interaction will have some Goal i.e. the objective that needs to be achieved using the interaction. In order to successfully achieve the goals of the interaction agent will need to go through the two stages namely Execution and Evaluation. The stages of an agent interacting with a MAS environment are described such that in order to accomplish a goal, which is in turn delegated to it by a human user, the following steps are traversed by an agent: Goal Formation, Intention formation, Action specification, Execution, Perception, Interpretation and Evaluation, as in the Action Cycle(AC) $[9,10]$. The essential concepts are the Gulfs of Execution and Evaluation, each arising as a result of semantic and articulatory distances (cognitive distances in general). As minimal architectural consequences, the elements necessary to achieve the design goals for the architecture through the AC Steps are shown in Figure 1 and described in Table 1.

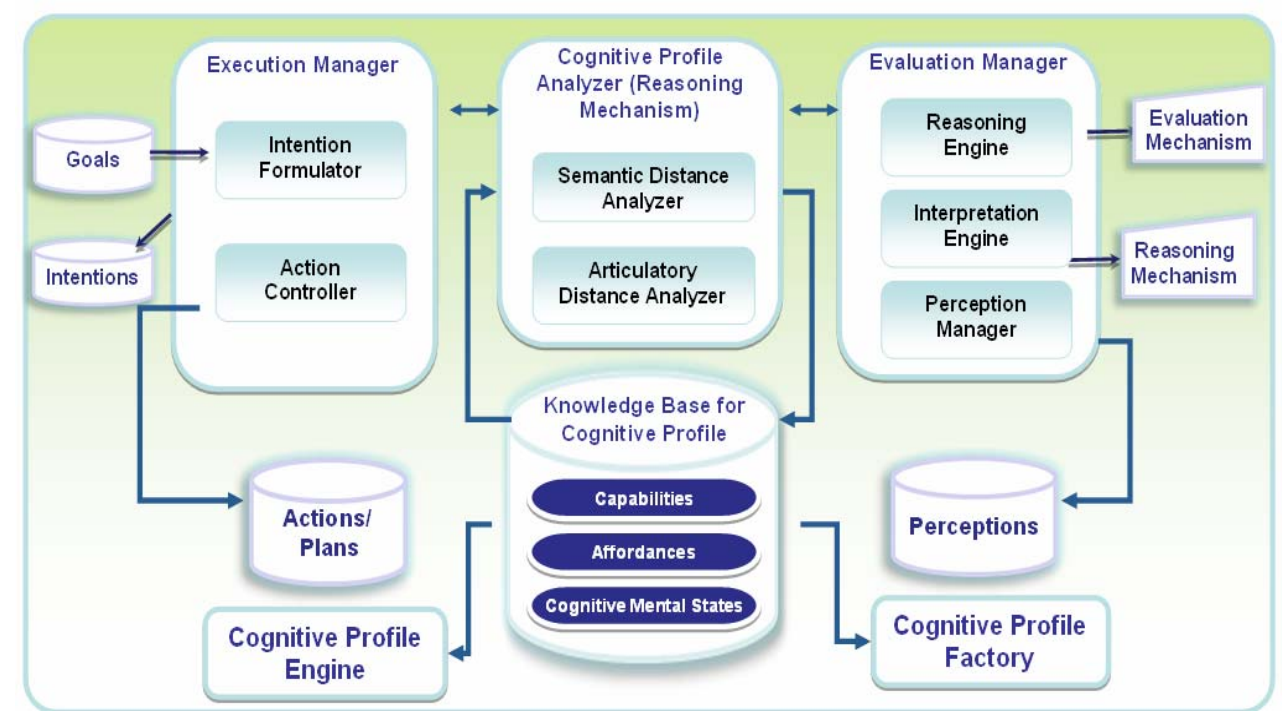

Figure 1: Infrastructure Elements in the Cognitive Profiling Architecture of Agents 
Table 1: Infrastructure Elements in the Cognitive Profiling Architecture of Agents

\begin{tabular}{l|l|}
$\begin{array}{l}\text { Infrastructural } \\
\text { Component }\end{array}$ & Description \\
$\begin{array}{l}\text { A } \\
\text { Representation } \\
\text { Mechanism } \\
\text { Prognitive }\end{array}$ & $\begin{array}{l}\text { Semantic representation mechanism needed that } \\
\text { enables the agents to discover and find out about } \\
\text { each others cognitive distances, semantic and } \\
\text { articulatory dispositions, capabilities, affordances } \\
\text { and constraints. For making the cognitive profile } \\
\text { available to agents }\end{array}$ \\
\hline $\begin{array}{l}\text { An Encoding } \\
\text { Mechanism }\end{array}$ & $\begin{array}{l}\text { For encoding the constructs of the cognitive profiles } \\
\text { i.e. the distances, affordances etc. }\end{array}$ \\
\hline $\begin{array}{l}\text { A Heuristic } \\
\text { Reasoning } \\
\text { Mechanism }\end{array}$ & $\begin{array}{l}\text { For making the agents reason about the parameters } \\
\text { of the cognitive profile both internal to their } \\
\text { architecture and external in their environment }\end{array}$ \\
\hline
\end{tabular}

\section{A Semantic Representation Model for Cognitive Profile of Agents}

At the heart of the architecture is the adoption of ontology to drive the cognitive profile of agents i.e. agent is given knowledge about its constraints and affordances, its semantic and articulatory disposition. From a philosophical perspective ontology can be defined as a set of things whose existence is acknowledged by a particular theory or system [12]. Such 'things' include both types (such as the class of Agents) and individual elements (such as the agent TravelAgent). The use of ontology is also prospective in the semantic web action space, since the emerging standards enable reasoning to be carried out effectively on such models using the heuristic reasoning rules defined; agent could then be made to reason about its state of processing. Architectures that facilitate the sharing of ontologies would enable the agents to discover each other's cognitive dispositions thus improving the manner in which they interact. The issue at stake is to be able to represent the cognitive profile adequately and in a manner that can be shared among agents. The Cognitive profile is therefore implemented as an Ontology in OWL, with OWL-DL (Description Logics) as basis of representation of the profile parameters and properties. Being an emergent standard, OWL-DL ensures that the model caters for a more open community. Ontologies have been recognised by the research community as a - model of expressing the knowledge model for agents e.g. by the recent research of [13].

\subsection{Conceptual Model for Agents' Cognitive Profile Ontological Model}

A generic conceptual model, with objects and properties of the ontological model to be implemented in order to enable shared cognitive profiling of agents is shown in Table 2 .

3.1.2. Cognitive Profile of Agent: The framework's correct implementation calls for maintaining a cognitive profile of semantic web agent. Following are the elements to be maintained in the cognitive profile: Agents Cognitive Mental States, Capabilities, Affordances, Semantic Disposition (Semantic Distances of Evaluation and Execution), Articulatory Disposition (Articulatory Distances of Evaluation and Execution), Goals, Intentions,
Perceptions, Evaluations. The mapping of the elements described in the conceptual model to OWL objects and properties is shown in Table 2.

3.1.2. Cognitive Mental State of an Agent: An agent modelled along the lines of the Cognitive $\mathrm{AC}$ requires various cognitive mental states corresponding to the various steps in the execution and evaluation stages of the action cycle to facilitate the agent's behaviour. It provides provision for state modelling, representation and tracking an agent's state of execution. In addition representing these states agents may have the dynamic capability of identifying these distances at runtime and may change and update their profile dynamically.

\subsection{OWL Model for Cognitive Profile of Agent}

The conceptual model is developed using the Protégé Ontology editor to generate OWL Ontology. Table 2 shows all the objects and properties that are part of the cognitive profile OWL model.

Table 2: Objects and Properties in the Generic Cognitive Profile Ontological Model

\begin{tabular}{|lll|}
\hline Object & Property & Object \\
\hline Agent & hasCapability & Capability \\
Agent & hasAffordance & Affordance \\
\hline Agent & hasNoCapability & Capability \\
\hline Agent & hasNoAffordance & Affordance \\
Agent & hasAgentID & AgentID \\
Agent & canAchieveGoal & Goal \\
Agent & canEvaluatePerception & Perception \\
Agent & hasDirectness & Directness \\
Agent & hasDistance & Distance \\
Agent & hasArticulatoryDistance & ArticulatoryDistance \\
Agent & hasCMS & CognitiveMentalState \\
Agent & hasGoal & Goal \\
Agent & canExpress & Intentions \\
Agent & canPerform & Action \\
Agent & canEvaluatePerception & Perception \\
Agent & canForm & Goals \\
\hline Agent & canAchieve & Goals \\
CognitiveMental & hasStage & Stage (Execution U \\
State(CMS) & & Evaluation) \\
\hline Distance & inExecutionStage & ExecutionStage \\
Distance & inEvaluationStage & EvaluationStage \\
Goal & requiresFulfilmentOf & Intention \\
Goal & requiresCapability & Capability \\
Goal & isEvaluatedBy & Evaluation \\
\hline Intention & isRealizedByAction & Action \\
Action & fulfillsIntention & Intention \\
Action & isRequiredForGoal & Goal \\
Action & requiresAffordance & Affordance \\
CMS & hasDistance & CognitiveDistance \\
CMS & hasDisposition & CognitiveDisposition \\
Perception & requiresAffordance & Affordance \\
\hline Evaluation & needsCapability & Capability \\
Evaluation & requiresInterpretationOf & Perception \\
\hline & & \\
\hline
\end{tabular}

\section{IMPLEMENTATION OF THE ARCHITECTURE}

Protégé ontology editor and Protégé OWL API [23, 24, $25]$ have been used for knowledge modelling, and the OWL ontology [27,28] for knowledge representation. The Jena library was used for knowledge and data manipulation, for querying and accessing cognitive profiles and the JADE agent system $[16,17]$ was used for real implementation of the communication scenario. The high-level architecture was evaluated using implementation of a travel planning scenario - customized adaptation from the vision of travel planning agents presented by [14] and later developed by [15]. The top level functional goals of the demonstration 
application are as follows: The Multi-Agent based application is aimed to use cognitively modelled agents to solve travel problems given by a user. The agents will Extract, filter and store information automatically from the semantic web using other agents. The system aims to use the same information that the user could find if he wish planning the travel himself. Cognitive Sharing of different kinds of abilities is to be demonstrated to gain efficiency in the problem solving task. The agents are simulated to reuse each others capacities, behaviours and offer affordances to each other. Agents closely work according to the user's characteristics, and functions based on the ultimate goals obtained from the user profile and adapt their functional behaviour according to the learned user preferences.

Reuse Mechanisms employed: The Behaviour API and Interaction Protocol API of JADE [16,17] are used to model the Agent's Actions, or an Action Plan. ACL Messages are used as representations for Intentions and perceptions. An agent is simulated in such a way that it is given runtime capability to change its behaviour, and dynamically change profile so as to demonstrate the power and potential of the Cognitive Profile Ontological Model.

Improved Cognitive Awareness: The provision of cognitive profile as shared knowledge base serves as means to increase the cognitive awareness for agents since they can not only reason about their own cognitive distances, they can also access and query other agents' cognitive profiles allowing them to adaptively refine their interactions in attempt to achieve their goals in a collaborative manner.

\subsection{Dynamic Sharing Mechanisms for Cognitive Profiles}

The cognitive profile ontology is central to representing the knowledge for agents. The elements of the cognitive profile ontology serve to represent the shared knowledge base of agents through which agents' cognitive awareness will be enhanced. The cognitive profiles are designed to simulate an environment such that some agents are limited in certain capabilities, while others are equipped with them in a complementary manner to facilitate interoperability, resuse and adaptive collaboration based on enhanced cognitive awareness.

4.1.1. Mechanism for Publishing Agents' Cognitive Profiles: Agents have automated, built in mechanism, such that as soon as they are created their profile is registered in the Shared Repository, or shared knowledge model. This has been achieved using the Protege OWL API [25] in combination with JenaOWLModel [18]. Using the API, the shared OWL model is accessed and updated by writing and saving the owl model. The process of publishing an agent's profile in the shared knowledge base is illustrated in Figure 2. It is assumed that the Cognitive Profile Knowledge Model is known at the design time, and is embedded by the designer. The defined elements of the profile are dynamically written into the profile upon agents' creation and removed upon its non existence or deletion or killing. Certain elements may be asserted at runtime.

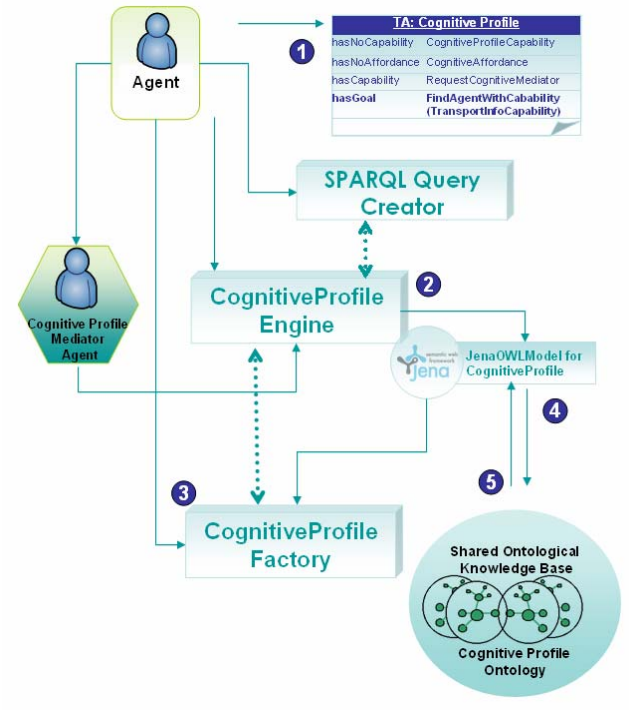

Figure 2: Implementation Architecture for Dynamic Sharing Mechanisms of Cognitive Profiles

As shown in the Figure 2, the possible actions that the agent performs to publish its profile are: (1)Get Profile Parameters of encoding the profile, (2) Get Reference to Profile Model using JenaOWLModel using the CognitiveProfile Engine (3) Use Cognitive Profile Factory to encode the Profile Parameters (4) Modify JenaOWLModel by adding a new OWL Individual using Objects and Properties (5)Save the Model . All these actions are automated (built-into) the agents' infrastructure.

4.1.2. Mechanism for Accessing the Shared Cognitive Profile Knowledge Base: There is no standardised way of accessing an OWL based shared knowledge base at the moment. However within the Semantic Web vision it could be assumed that: The ontologies are available within a network - they may have different access rights - but given an agent, it would have access to certain ontologies i.e the Cognitive Profile Ontology in this case. The Semantic Web would need to be indexed in order to be searchable. In the current Web search engines index the Web. In the Semantic Web there may be similar intermediaries. Here, the Cognitive Profile Mediator Agent (shown in Figure 2) plays this role of an intermediary. If an agent wanted to obtain specific information and/or execute specific behaviour, it would need to query the semantic engine/intermediary to obtain the results (e.g., a list of sources that could supply the information/behaviour), Select among the sources returned, Combine and orchestrate the queries/invocations to the chosen sources and Receive and aggregate the results.

Taking above considerations into account, it is assumed that all agents may or may not have full knowledge of the Cognitive Profile Ontologies. It is also assumed that for the sake of simplicity, that there is only once centralized knowledge base (ontology) in which all agents' cognitive profiles are maintained. In reality however it is unrealistic that an agent would be aware of a resource unless it has been 
previously indexed by some intermediary. A hybrid approach has been adapted. Some agents have public/open access to the cognitive profile. Those who do not will be able to do so using the Cognitive Profile Mediator Agent to get the required profiles. SPARQL [26] has been used to query the ontology since JENA enables querying an ontology with SPARQL. For instance if an agent wants to find another agent with a desired cognitive profile, it uses the Query Creator to pass their desired query to the Cognitive Profile Mediator Agent, which uses the Cognitive Profile Engine (shown in Figure 2) which in turn uses JenaOWLModel to find the QuerySolution in the form of Resources and Literals. The results obtained from the CP Knowledge Base are interpreted and filtered using the Cognitive Profile Engine and returned to the respective agents.

\subsection{Implementation Details of Heuristic Reasoning Mechanisms}

4.2.1. Abstract Heuristic Reasoning Mechanism for Agents: To bridge the gulfs of execution and evaluation, agent must have some heuristic reasoning mechanism built into its architecture, such that given a shared cognitive profile is available, it should be able to reason on the knowledge present in it to aid the agent's processing and help bridge the gulfs of execution and evaluation through collaboration or other means. The important considerations are with regards to the representation, discovery and reasoning of the cognitive distances. Rules generate advice by defining the combination of agent knowledge, action stages, distances, abilities/capabilities, and affordances.

4.2.2. Pre-Classification Of Cognitive Profiles Using Reasoner A Reasoner may be invoked e.g. Racer to preclassify agent's semantic and articulatory disposition with respect to certain scenarios. Agents are classified into subclasses based on necessary and sufficient conditions defined. This allows for richer cognitive awareness. Rules may be implemented as necessary and sufficient condition in the protégé owl ontology and the reasoner can pre-classify agents based on their capabilities etc. JENA Reasoner or any other reasoner may also be used [18].

4.2.3. SWRL-JESS Rule Based Reasoning: The Ontology is queried using the SPARQL Query language [26]. These rules are implemented in SWRL [22] using the Protégé SWRL Plugin [24]. Rules are defined to reason about agent's cognitive mental states and Semantic and Articulatory disposition. Facts may be asserted at run time and reasoning carried out using an expert system such as JESS (Java Expert System Shell) [19, 20]. A derivative of it such as OWLJessKB[30] may also be used. A small scenario using some SWRL Rules in combination with JESS Bridge has been used to demonstrate this. Any explicit reasoning mechanism may be used. The dynamic assertion also comes into play especially when reasoning about the agents semantic and aritculatory disposition in its execution and evaluation stages.

\section{The USE AND Rationale Behind Cognitive Profiling OF AgENTS}

The framework is intended to aid the designer in modelling the agent behaviour and action through a cognitive cycle. The cognitive distance models allows for limitations in agents semantic capabilities and affordances be identified and maintained in a cognitive profile which can then be shared with other agents. Agents that normally exist are pre-defined in their attributes and capabilities and can only perform the functions they perform. If the mental models of agents were to map with each other, using this model, their capabilities could in return complement each other. Agents may delegate each other tasks and goals based on each other's awareness of abilities. So less cognitive overload is incurred on humans. Modelling the agent's interaction using the action cycle, identifying the gulfs of evaluation and execution through the cognitive distances (semantic and articulatory) allows gaps to be identified, providing for a more robust design of agent applications. The cognitive modelling of the agent's execution model allows for a more dynamic, pro-active and vibrant design, one that is able to adapt to the user goals to a varying degree. An important implication of Cognitive Profiling Architecture is towards developing agents whose intrinsic capabilities and architecture facilitate interoperation amongst heterogenous agents. Agents are able to carry out cognitively aware communication and collaboration with each other. Agents are not only able to cognitively describe, publish and access their services but also capabilities, affordances and distances/constraints in an agent's internal structure allowing for a more dynamic and adaptive behaviour of agents on the semantic web. Thus the enhanced cognitive awareness improves the level of negotiation and collaboration between agents

\section{Conclusions AND Future REsearch DIRECTIONS}

The implications of cognitive models adapted from Cognitive sciences are presented in this paper within a semantic web enabling infrastructure and provided an infrastructure for agents, including abstract and discrete models for Cognitive Profiling and Heuristic Reasoning. The application not only validates the architecture but also illustrates the adaptive coordination of different agents acting as owners in heterogenous and dynamically changing environments. The results of the evaluation show an improved flexibility, interoperability and reusability of agents' collective behaviours and goals. Thus, it establishes the Cogntive Profiling Architecture for agents as a step forward in providing the next generation of Semantic Web, a successful framework of multi-agent collaboration, which is inevitably required for generating robustly engineered agents able to carry out spontaneous and adaptive 
collaboration based on cognitive awareness of their environment and infrastructure.

The abstract architecture presented here opens new doors of research. With the core framework in place the natural next step in its expansion is the specialised enhancement towards a more rigorous definition of different levels and variations of cognitive profile and its parameters. Identifying the best level of detail for functionally decomposing each task or intention and applying it consistently is difficult. A serious issue in making this model work on a larger scale will be ensuring the standardization for the interoperability. Issues of Ontology learning [31], Ontology alignment and mapping (as highlighted in works by $[32,33]$ ) also become important for standardization and homogenization purposes. Standardization of cognitive profile for agents will be another issue foreseen if this model were to work successfully, but with rich semantic model of OWL and $\mathrm{RDF}$ will allow for standardization to be achieved. However it provides substantial stimulus for future research.

\section{REFERENCES}

1. Jennings N. R. 2000, "On agent-based software engineering". Artificial Intelligence, vol. 117, pp. 277-296.

2. Jennings, N.R., Sycara, K. \& Wooldridge, M. 1998.b, "A Roadmap of Agent Research and Development", Autonomous Agents and MultiAgent Systems, vol. 1, no. 1, pp. 7-38.

3. Wooldridge, M.J., 2002. An Introduction to Multi-Agent Systems. John Wiley \& Sons.

4. Wooldridge, M. \& Jennings, N.R. 1995, "Intelligent agents: Theory and practice", The Knowledge Engineering Review, vol. 10, no. 2, pp. 115-152.

5. Louis, V., Martinez, T., 2005a, "The Jade Semantic Agent: Towards Agent Communication Oriented Middleware", AgentLink News Journal, no. 18, pp.16-18.

6. Louis V., Martinez T., 2005b, “An operational model for the FIPAACL semantics". Proceedings of the AAMAS'05 Workshop on Agent Communication (AC2005), Utrech, The Netherlands.

7. Sun, R. 2001, "Cognitive science meets multi-agent systems: A prolegomenon", Philosophical Psychology, vol. 14, no. 1, pp. 1-28.

8. Willmott, S. (ed.), 2003 "Technical Input and Feedback to FIPA from Agentcities.RTD and the Agentcities Initiative". Agentcities Task Force Technical Note 00003, Available from: http://www.agentcities.org/note/00003/

9. Norman, D. A. 1992, "Design Principles for cognitive artefacts", Research in Engineering: Design Theory Applications and Concurrent Engineering, Springer Verlag, vol. 4, no. 1, pp. 43-50.

10. Norman, D. A. 1986, "Cognitive engineering", In: Norman, Donald A., Draper, Steven (Eds.) "User Centered System Design: New Perspectives on Human-Computer Interaction". Lawrence Erlbaum Associates

11. Norman, D.A., Draper, S., 1986 "User Centered System Design: New Perspectives on Human-Computer Interaction". Lawrence Erlbaum Associates

12. Bell, D., De Cesare, S., Iacovelli, N., Lycett, M. \& Merico, A. 2007, "A framework for deriving semantic web services", Information Systems Frontiers, vol. 9, no. 1, pp. 69-84.

13. Laclavík, M., Balogh, Z., Babík, M. \& Hluchý, L. 2006, "Agentowl: Semantic knowledge model and agent architecture", Computing and Informatics, vol. 25, no. 5, pp. 421-439.

14. Hendler, J. 1999, "Is there an intelligent agent in your future?", Nature.

Available www.nature.com/nature/webmatters/agents/agents.html

15. Camacho, D., Borrajo, D. \& Molina, J.M. 2001, "Intelligent Travel Planning: A MultiAgent Planning System to Solve Web Problems in the e-Tourism Domain", Autonomous Agents and Multi-Agent Systems, vol. 4, no. 4, pp. 387-392.
16. JADE, 2004a, JADE (Java Agent DEvelopment Framework) [Internet] Available from: http://jade.cselt.it/[Accessed: 20th July 2007]

17. JADE, 2004b, JADE Documentation, [Internet] Available from: http://jade.cselt.it/[Accessed: 20th July 2007]

18. JENA, HP Labs and Open Source Community, 2006, "Jena - A Semantic Web Framework for Java", [Internet] Available from: http://jena.sourceforge.net/[Accessed: 20th July 2007]

19. Friedman-Hill, E.J. 1999, "Jess, the Java Expert System Shell", Available from: http://herzberg.ca.sandia.gov/jess; [Accessed: 20th July 2007]

20. Friedman-Hill, E., 2003, "JESS In Action: Rule-Based Systems in Java", Manning Publications, Greenwich, CT.

21. FIPA, 2002, Foundation for Intelligent \& Physical Agents (FIPA) Agent Communication Language specification. [Internet] Available from: http://www.fipa.org/repository/aclspecs.html [Accessed: 20th July 2007]

22. O'Connor, M., Knublauch, H., Tu, S., Grosof, B., Dean, M., Grosso, W. \& Musen, M. 2005, "Supporting rule system interoperability on the semantic Web with SWRL", Lecture Notes in Computer Science (Artificial Intelligence and Lecture Notes in Bioinformatics), vol. 3729 LNCS, pp. 974-986.

23. SMI (Stanford Medical Informatics Group), 2007a., "Protégé ontology editor and knowledge-base framework" [Internet] Available from: http://protege.stanford.edu/[Accessed: 20th July 2007]

24. SMI (Stanford Medical Informatics Group), 2007b., "Protégé-OWL" [Internet] Available from: http://protege.stanford.edu/overview/protege-owl.html [Accessed: 20th July 2007]

25. SMI (Stanford Medical Informatics Group), 2007c., "Protégé OWL API" [Internet] Available from: http://protege.stanford.edu/plugins/owl/api/index.html [Accessed: 20th July 2007]

26. W3C, 2007, "SPARQL Query Language for RDF -W3C Candidate Recommendation"[Internet] Available from: http://www.w3.org/TR/rdf-sparql-query/ [Accessed: 20th July 2007]

27. W3C, 2004a, "W3C: Web Ontology Language (OWL) Overview". [Internet] Available from: http://www.w3.org/TR/owl-features/ [Accessed: 20th July 2007]

28. W3C, 2004b, W3C: Resource Description Framework RDF. [Internet] Available from: http://www.w3.org/RDF/[Accessed: 20th July 2007]

29. W3C, 2004c, "OWL Web Ontology Language Guide- W3C Recommendation " [Internet] Available from: http://www.w3.org/TR/owl-guide/[Accessed: 20th July 2007]

30. OWLJessKB, 2005, "OWLJessKB: A Semantic Web Reasoning Tool" [Internet] Available from: http://edge.cd.drexel.edu/assemblies/software/owljesskb/ [Accessed: 20th July 2007]

31. Maedche, A. \& Staab, S. 2001, "Ontology learning for the semantic web", IEEE Intelligent Systems and Their Applications, vol. 16, no. 2, pp. 72-79.

32. Sampson, J. \& Lanzenberger, M. 2006, "Visual ontology alignment for semantic web applications", Lecture Notes in Computer Science (Artificial Intelligence and Lecture Notes in Bioinformatics), vol. 4231-LNCS , pp. 405-414.

33. Laera, L., Tamma, V., Euzenat, J., Bench-Capon, T. \& Payne, T. 2006, "Reaching agreement over ontology alignments", Lecture Notes in Computer Science (Artificial Intelligence and Lecture Notes in Bioinformatics), vol. 4273-LNCS , pp. 371-384. 